O5 (continued)

Funding: Illinois State University College of Applied Science \& Technology University Research Grant

\section{Association Between Healthful Eating Behaviors and Intentions to Feed Future Children Healthfully Among College Students}

Jen Nickelson, PhD, RD, jnickelson@ches.ua.edu, University of Alabama, Box 870311, Tuscaloosa, AL 35487; Mary Nelson Robertson, MS, Mississippi State University

Objective: The Theory of Planned Behavior proposes that the immediate antecedent to a behavior is the intention to perform that behavior. Hence, if parents plan (intend) to feed their children healthfully, they will be more likely to do so. Parents' child feeding intentions (CFI) may be associated with their own eating behaviors, as parents' eating behaviors have been correlated with children's eating behaviors. The purpose of this study was to examine the association between CFI and healthful eating behaviors among childless adults who plan to have children.

Study Design, Setting, Participants: Using a crosssectional study design, we surveyed 421 undergraduate students enrolled in randomly-selected undergraduate courses. Complete, valid data for all variables were provided by 297 students who plan to have children.

Outcome Measures and Analysis: The outcome variable was an indicator of CFI derived from an instrument designed to assess intentions to use Satter's division of responsibility (sDOR-I) in child feeding (psychometric properties described elsewhere). Indicators of healthy eating were fruit and vegetable (FV) consumption and eating competence (EC). Multiple linear regression was used to analyze the association among students' CFI, FV intake, EC, gender and age.

Results: The model accounted for $18.4 \%$ of the variance in CFI $[\mathrm{F}(4,291)=16.44, \mathrm{p}<.000, \mathrm{R} 2=.184]$. Controlling for other variables in the model, FV intake, gender (female) and age were significantly and positively associated with CFI.

Conclusions and Implications: This study is a first step in beginning to understand CFI among people who do not yet have children. Having this understanding may be useful in improving child feeding behaviors.

Funding: None

\section{Investigating the Preliminary Effects of Little Foodies: A Health Promotion Program for Parents of Toddlers}

TraceyLedoux,PhD,RD,FAND, taledoux@uh.edu, University of Houston, 3875 Holman Street, Garrison Gym Room 104, Houston, TX 77204-6015;

Stephanie Silveira, BS, University of Houston; Jamie Le; Hiba Kamal, BS; Stephanie Kung
Objective: Obesogenic eating behaviors are seen in children as young as 3-5 years of age. Parent feeding practices prevent or promote obesogenic eating behaviors. The purpose of this study was to pilot test a parent focused intervention aimed at promoting healthy eating behaviors among toddlers (12-48 months).

Outcome Measures and Analysis: Parents were recruited from the community to participate in Little Foodies (LF), which consisted of six weekly classes with instruction, discussion, video feedback, goal setting, and refreshments. Measures of parent feeding practices and child eating behaviors were collected pre and post. A satisfaction survey and focus group were conducted post. Process variables were tracked. Paired sample t-tests were conducted on outcomes. Significance was set at $\mathrm{p}<.10$ given the exploratory nature of this study.

Results: Twenty-two primarily Caucasian (55\%) mothers (91\%) from middle to upper socioeconomic conditions (63\% college educated, $77 \%$ married, $77 \%$ earn $>\$ 50 \mathrm{~K}$ ) participated. LF had preliminary effects on indulgent feeding $(\mathrm{p}=.03 ; \mathrm{d}=.47)$, caregiver distress at meals $(\mathrm{p}=.06$; $\mathrm{d}=.43)$, parent control $(\mathrm{p}=.10), \mathrm{d}=.52)$, toddler picky eating $(\mathrm{p}=.08, \mathrm{~d}=.29)$, and toddler control $(\mathrm{p}=.07$; $\mathrm{d}=.40$ ); but no effects on responsive feeding, forceful feeding, restrictive feeding, uninvolved feeding, and child social behaviors at meals $(\mathrm{p}>.10)$. In focus groups participants reported that they learned from other parents, they felt less reactive at meals, and they had more confidence in responsive feeding. On satisfaction surveys, $87-93 \%$ of participants enjoyed and valued LF "very much" and learned "very much" from LF.

Conclusions and Implications: The preliminary evidence of LF suggests it is a promising, feasible health promotion program for parents of toddlers. Future research on a larger, more diverse sample with a control group is warranted.

Funding: University of Houston

\section{When Kids Learn to Cook: Findings from the Illinois Junior Chefs Effectiveness Trial}

Jessica Jarick Metcalfe, MPH, jarick2@illinois.edu, University of Illinois at Urbana-Champaign, 904 West Nevada Street, MC-081, Urbana, IL 61801;

Barbara Fiese, PhD, University of Illinois at UrbanaChampaign; Ruyu Liu; Emillie Emberton; Jennifer McCaffrey, PhD, MPH, RD

Objective: Assess the effectiveness of the Illinois Junior Chefs (IJC) program in generating positive changes in participants' dietary attitudes and behaviors.

Target Audience: SNAP-Ed eligible youth $(\mathrm{n}=1161)$ aged 8-14 (average age $=9.6$ ).

Theory, Prior Research, Rationale: Recent evidence indicates that the majority of children in America do not meet federal nutrition guidelines. Research indicates that participation in cooking is associated with healthier dietary intake in children and adolescents, therefore experts advocate for increased hands-on culinary education for this age group. 
O8 (continued)

Description: IJC is a statewide cooking and nutrition education program implemented in summer 2016 by the University of Illinois Office of Extension and Outreach. Over the course of five 2-hour lessons, youth participated in hands-on culinary skill building activities, nutrition education, recipe preparation, and healthy food tastings. Lessons took place in varied community settings, examples include summer camps, libraries, churches, community centers, and Extension offices.

Evaluation: Analyses of pre- and post-intervention surveys using paired t-tests indicated that participants experienced significant pre- to post-intervention changes in cooking self-efficacy $(\mathrm{t}(1153)=18.85$, $\mathrm{p}<.001)$, fruit and vegetable preferences $(\mathrm{t}(1132)=4.48$, $\mathrm{p}<.001)$, cooking attitudes $(\mathrm{t}(1125)=9.77, \mathrm{p}<.001)$, cooking behaviors $(\mathrm{t}(1099)=3.79, \mathrm{p}<.001)$, and self-efficacy for selecting and eating healthy foods $(\mathrm{t}(1091)=$ 7.71, $\mathrm{p}<.001$ ). Researchers also observed classes at a subset of sites and interviewed nutrition educators to assess fidelity, facilitators and barriers to program implementation, and gain insights for future curriculum modifications.

Conclusions and Implications: The IJC program resulted in significant improvements in participants' dietary attitudes and behaviors. These program effects could result in positive long-term impacts on participants' dietary health, and contribute to the evidence base supporting the efficacy of the IJC program.

Funding: Supplemental Nutrition Assistance Program Education USDA NIFA 2011-67001-30101; 4-H Foundation

\section{A Digital Approach to Behavior Change - Helping Low-Income Moms to Shop, Cook, and Eat Healthy on a Budget}

Kim Laramy, BS, klaramy@ethos-marketing.com, Ethos Marketing, 17 Ash Street, Westbrook, ME 4092

Objective: Learn how a digital approach to reaching Maine moms helped them to shop, cook, and eat healthy on a budget.

Target Audience: The campaign employed detailed targeting analysis to find and reach low-income moms and serve up easy, relevant tips and tricks.

Theory, Prior Research, Rationale: Focus group learning pointed to the fact that smartphones are low-income Moms' primary connection to the world, the way they get information and access services. Moms also shared that they want to feed their families healthy, but aren't sure how to and perceive it to be expensive.

Description: A campaign and developed an integrated approach including a content-rich, mobile-friendly website, direct mail, a digital campaign with real moms sharing their tips. Digital outreach included keyword search campaigns, digital display campaigns, YouTube videos, and content development and promotion on Facebook.
Evaluation: Independent evaluation confirmed that in one year $81 \%$ of participants reported being confident in their ability to buy healthy foods on a budget for their family, and there was a $14 \%$ increase in SNAP-Ed program participants from the previous year. The website logged average monthly impressions of over 1.5 million and logged average clicks to the website of over 3,800 per month. A Facebook campaign was launched and grew to over 2,700 fans in just over a year with a high level of engagement.

Conclusion and Implications: Social media created an engaging campaign geared directly to low-income moms. Funding: None

\section{Does Hands-On Cooking Instruction Before Adulthood Support Healthy Food Behaviors to Become Lifestyle Choices?}

Anjuman Shah, MS, Columbia University; Pam Koch, EdD, RD; Casey Wilson, MS, FamilyCook Productions; Lynn Fredericks, BA, lynn@familycookprodu ctions.com, FamilyCook Productions, 330 East 43rd Street, Suite 704, New York, NY 10017

Objective: This study examines alumni of Teen Battle Chef (TBC), a hands-on culinary program, for its impact on sustaining positive eating behaviors.

Target Audience: Qualitative interviews with 30 TBC alumni, 1-7 years post TBC, who described learning to cook in TBC and how program components supported them in making and sustaining dietary changes.

Theory, Prior Research, Rationale: TBC was designed using SCT and Social Ecological Theories. Prior research on TBC found improvements in food choices, level of physical activity, empowerment to share with others, and increased self-efficacy to prepare healthy meals.

Description of Course and Curriculum: TBC introduces culinary skills by having two teams "battle" each other in TV-like cooking demos, while exploring health benefits of each recipe. Students develop leadership qualities and skills and can move onto summer internships cooking at farmers markets, gardens, preschools, restaurants and caterers.

Evaluation: Interviews were recorded, transcribed, and coded using NVivo to identify a range of themes that propelling interviewees to change their habits. Eleven total themes ranged from adventurous eating, peer/family influence, personal weight loss, family/friends weight loss, and most influential skills acquired, to increased self-confidence/esteem and following the dietary guidelines.

Conclusions and Implications: Analysis of alumni experiences indicates strong links between development of basic culinary skill to high self confidence and self actualization. Most participants expressed satisfaction in making dietary changes, losing weight themselves, and supporting friends and family members to do the same. Participants shared their own realization that healthful food can be easy to prepare and delicious and how it became "self 